\title{
Erratum to: Reentrant Superconductivity in $\mathrm{UTe}_{2}$
}

\author{
V. P. Mineev ${ }^{a, b}, *$
}

${ }^{a}$ Univ. Grenoble Alpes, Commissariat a l'Energie Atomique, PHELIQS, GT, Grenoble, F-38000 France

${ }^{b}$ Landau Institute for Theoretical Physics, Russian Academy of Sciences, Chernogolovka, Moscow oblast, 142432 Russia *e-mail: vladimir.mineev@cea.fr

Received January 27, 2021; revised January 27, 2021; accepted January 27, 2021

DOI: $10.1134 / \mathrm{S} 0021364021010124$

Page 718, Eqs. (21-23) should read as follows:

$$
\begin{gathered}
\frac{e \hbar}{m_{0} c} H_{c 2}^{\mathrm{lin}}(0) \approx \frac{10 \hbar}{V_{\mathrm{F}} d m_{0}} \frac{T_{c}^{2}}{t}, \\
t \lesssim \frac{\hbar}{V_{\mathrm{F}} d m_{0}}(\text { Kelvin }), \\
H>H_{0}=\frac{8 \hbar^{2}}{\left(V_{\mathrm{F}} d m_{0}\right)^{2}} \text { (Tesla) } .
\end{gathered}
$$

The original article can be found online at https://doi.org/10.1134/S0021364020120036 